\title{
Hierarchical self-assembly of suspended branched colloidal nanocrystals into superlattice structures
}

\author{
Karol Miszta ${ }^{1 \dagger}$, Joost de Graaf ${ }^{2 \dagger}$, Giovanni Bertoni ${ }^{1 \dagger}$, Dirk Dorfs ${ }^{1}$, Rosaria Brescia ${ }^{1}$, Sergio Marras ${ }^{1}$, \\ Luca Ceseracciu' ${ }^{1}$, Roberto Cingolani ${ }^{1}$, René van Roij ${ }^{3}$, Marjolein Dijkstra ${ }^{2}$ and Liberato Manna ${ }^{1 \star}$
}

\begin{abstract}
Self-assembly of molecular units into complex and functional superstructures is ubiquitous in biology. The number of superstructures realized by self-assembly of man-made nanoscale units is also growing. However, assemblies of colloidal inorganic nanocrystals ${ }^{1-3}$ are still at an elementary level, not only because of the simplicity of the shape of the nanocrystal building blocks and their interactions, but also because of the poor control over these parameters in the fabrication of more elaborate nanocrystals. Here, we show how monodisperse colloidal octapod-shaped nanocrystals self-assemble, in a suitable solution environment, on two sequential levels. First, linear chains of interlocked octapods are formed, and subsequently the chains spontaneously self-assemble into threedimensional superstructures. Remarkably, all the instructions for the hierarchical self-assembly are encoded in the octapod shape. The mechanical strength of these superstructures is improved by welding the constituent nanocrystals together.
\end{abstract}

The organization of colloidal nanocrystals into ordered structures is a necessary step towards the fabrication of artificial solids and new devices. Superstructures can be built either by self-assembly directly in solution, or on a substrate following solvent evaporation or de-wetting ${ }^{4-6}$. A variety of forces can be involved in their formation: van der Waals (vdW) attractions between the particles, steric repulsions between the hydrophobic tails of the surfactants (often coating the nanocrystal surface), capillary forces during solvent evaporation, attractive depletion forces, Coulomb forces between surface charges or electric dipoles, and magnetic forces ${ }^{1,3,5,7-12}$. The assembly of many ordered threedimensional (3D) superstructures, for example, simple, binary, or ternary assemblies of spherical nanoparticles ${ }^{13-17}$, and smectic-like multilayers of hexagonally packed nanorods ${ }^{18}$, as well as liquid crystalline phases, is found to be solely driven by entropy ${ }^{19-21}$. More elaborate assemblies could be achieved from such simple building blocks by encoding information for the self-assembly in the surface pattern of the nanoparticles, for instance by DNA functionalization to modify the strength and directionality of particle-particle interactions $\mathrm{s}^{22-24}$. Furthermore, bifunctional linkers and key-lock molecular pairs have been employed to align nanorods in chain-like structures ${ }^{25}$. Directional electric and/or solvophobic interactions were further employed to drive the organization of spherical nanoparticles into lattices ${ }^{26}$. Finally, templating has been successfully applied to create hierarchical superstructures using principally spherical particles ${ }^{27,28}$, and layerby-layer methods have been exploited to generate hierarchically organized material $s^{29}$. However, limitations in synthesis, selective functionalization, and purification processes have posed serious
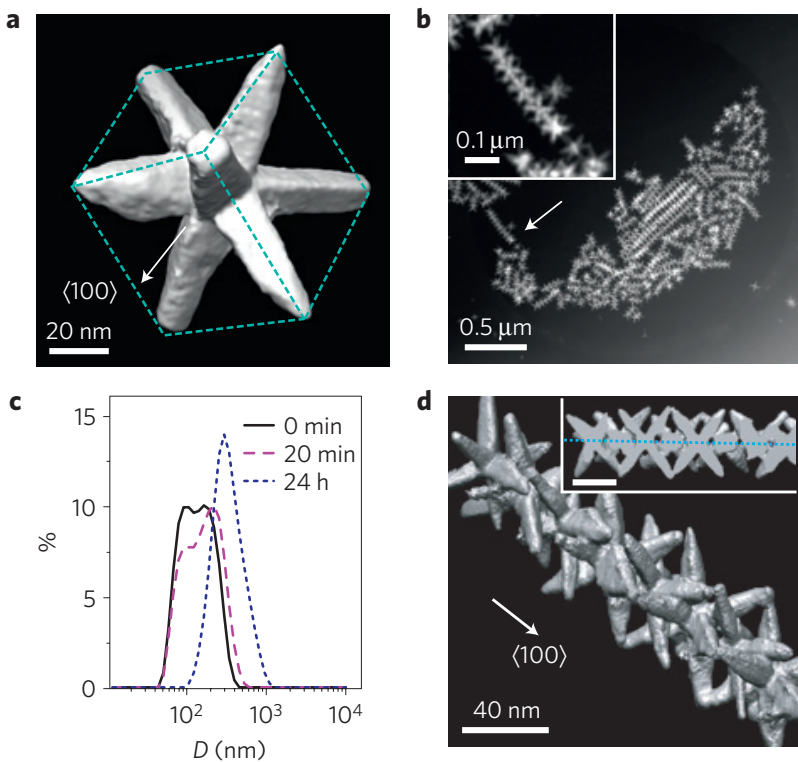

d

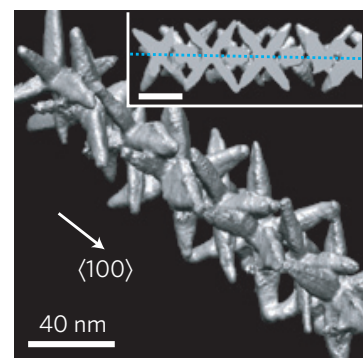

Figure 1 | Formation of octapod chains in toluene. a, 3D reconstruction of a single branched nanocrystal (octapod) from STEM projections, revealing an octahedral symmetry (the dashed lines mark the cube spanned by the tips of the octapod). $\mathbf{b}$, STEM image from the vitrified toluene solution after approximately $24 \mathrm{~h}$ of ageing, revealing the presence of $\sim 0.4 \mu \mathrm{m}$ long chains of octapods. The inset shows a magnified view of a single chain. c, DLS cluster size distribution curves from the toluene solution at different ageing times, confirming the formation of aggregates (the chains). $D$ is the size of the aggregates, $\%$ is the percentage frequency. $\mathbf{d}, 3 \mathrm{D}$ reconstruction of a single chain of octapods, demonstrating their interlocking sequence along $\langle 100\rangle$ of the cube in $\mathbf{a}$, with the octapod centres well aligned on the chain axis, as shown by the cut-through of the inset

limits to the quality and reproducibility of such assemblies and to their maximum attainable size.

Branched nanocrystals such as tetrapod or octapod-shaped colloidal nanoparticles have recently emerged as promising materials for photovoltaics and electronics ${ }^{27,28,30,31}$, and questions have been raised on how to approach their spatial organization. Compared to the simple nanocrystals described above, the assembly of branched nanocrystals poses several further challenges. First, it has not been possible until now to synthesize samples of branched nanocrystals with a sufficiently monodisperse distribution of size and shape, as well as with enough stability in the solution phase, to systematically

${ }^{1}$ Istituto Italiano di Tecnologia (IIT), Via Morego 30, 16163 Genova, Italy, ${ }^{2}$ Soft Condensed Matter, Debye Institute for Nanomaterials Science, Utrecht University, Princetonplein 5, 3584 CC, Utrecht, The Netherlands, ${ }^{3}$ Institute for Theoretical Physics, Utrecht University, Leuvenlaan 4, 3584 CE, Utrecht, The Netherlands. †These authors contributed equally to this work. *e-mail: liberato.manna@iit.it. 

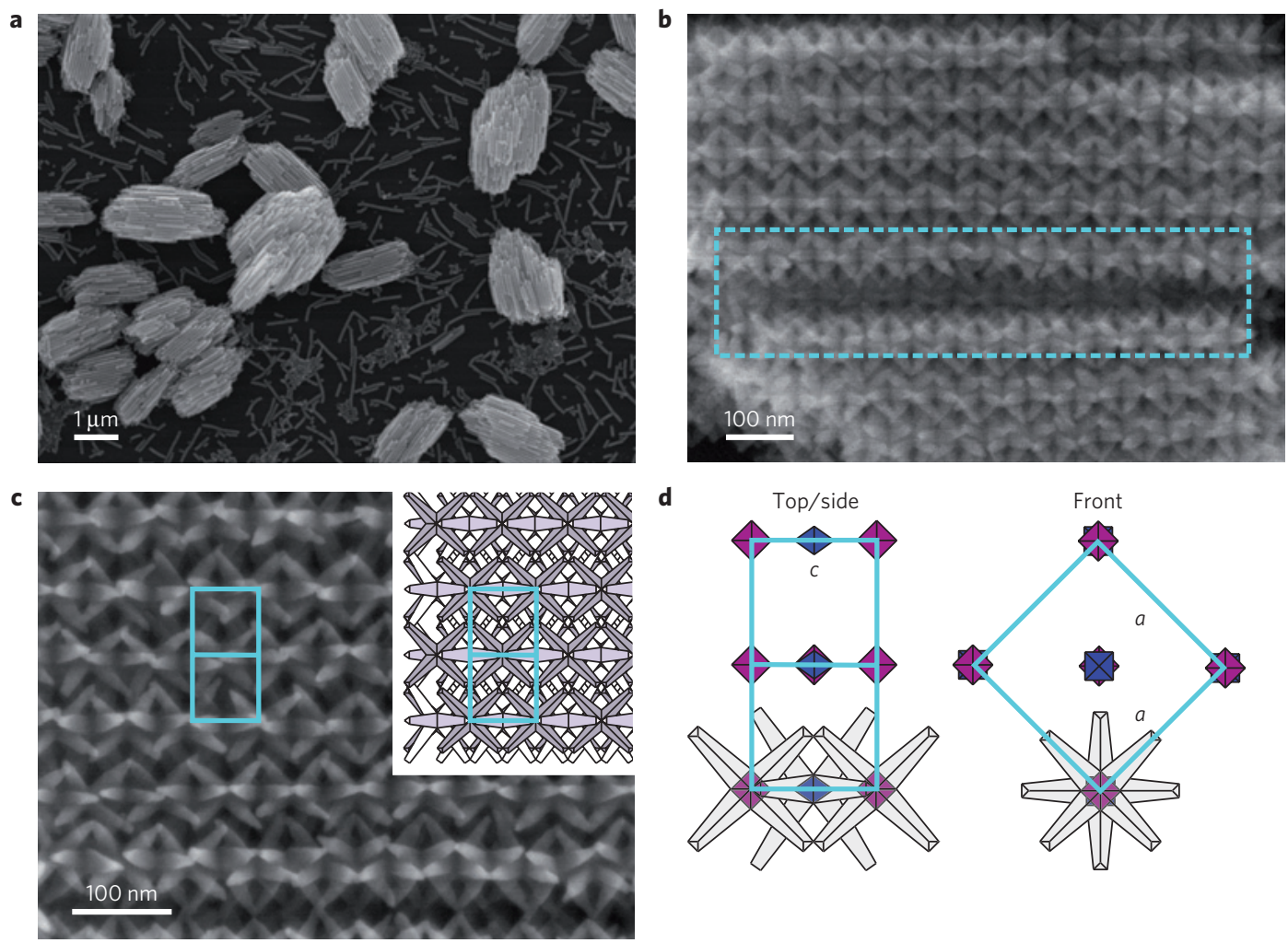

Figure $\mathbf{2}$ | Assembly of chains into 3D superstructures. a, After the addition of acetonitrile to an aged solution of octapods in toluene, 3D clusters are formed, as revealed by SEM after deposition on a silicon substrate. $\mathbf{b}$. The clusters are $\sim 2 \mu \mathrm{m}$ long and are formed by ordered packing of pre-formed chains (the cyan box marks a missing chain in the structure, showing the orientation of the layer underneath). c, Close view of the structure in a cluster, matching a tetragonal structure (sketched in the inset). d, Model of the tetragonal unit cell of the superstructure, as seen from top/side and front views (with respect to the silicon substrate), $a$ and $c$ being the base edge and height of the unit cell, respectively, with $a \approx c \sqrt{ } 2$, and $c$ approximately $70 \pm 3 \mathrm{~nm}$. The $c$ axis is oriented as the chain direction (blue and magenta octahedrons stand for the two orientations of an octapod in the interlocking sequence of the chain). See Supplementary Information for details.

investigate their assembly. Second, the complex shape of these particles should considerably restrict the rotational and positional degrees of freedom in neighbouring nanoparticles at high volume fractions, which may inevitably trap the crystallization process in 'jammed' amorphous structures. On the other hand, the inherent shape of the nanocrystals might already encode the information required to direct their assembly along a preferred path, under suitable experimental conditions. Third, compared with spherical, rod-shaped, or polyhedral particles, many densely packed configurations favoured by excluded-volume interactions might exist for branched nanoparticles. These configurations, aside from being kinetically difficult to achieve, may be differently affected by other energy contributions (for example, vdW, electrostatic, dipolar), making both their experimental realization and computer simulation daunting tasks.

Our group has recently overcome all these problems and developed a synthesis scheme to create octapod-shaped nanocrystals, in which eight CdS 'pods' are grown from a central region made of CdSe (ref. 31). Further refinements of the synthesis (see Methods and Supplementary Information) have allowed us to prepare octapods with unprecedented shape homogeneity and monodispersity (see for instance Supplementary Fig. S2, and the 3D reconstruction of one octapod in Fig. 1a). These nanocrystals are coated with hydrophobic surfactant molecules and therefore they favourably interact with hydrophobic solvents, that is, non-polar or moderately polar solvents.

We will limit the discussion to the $\mathrm{vdW}$ interactions, as they are predominant in the present case. We observed that octapods dissolved in chloroform remain stable and separated in solution, as confirmed by both dynamic light scattering (DLS) and cryo-scanning transmission electron microscopy (STEM) (Supplementary Fig. S1), thereby suggesting that the octapod-chloroform attractive interactions are on average of the same strength as the octapod-octapod interactions, or stronger. Interestingly, when the octapods were dissolved in toluene, they slowly started aggregating, on a timescale of a few hours. Apparently, the octapod-toluene attractions are slightly weaker than the octapod-octapod interactions in toluene. The aggregation could be followed by DLS (Fig. 1c), or again by cryo-STEM, as seen in Fig. 1b, which shows an image of an aliquot taken after $24 \mathrm{~h}$ of ageing. Here, although some isolated octapods were still present in the solution, most of them were arranged in chain-like assemblies. To further investigate the self-organization, we deposited a drop of this solution on a standard carbon-coated TEM grid. After solvent evaporation, many isolated chains were found on the carbon support. A STEM tomographic reconstruction revealed that every octapod in the chain is 'interlocked' with its two neighbouring octapods (Fig. 1d and inset). No other types of superstructures beyond the interlocked chains were present in toluene (see Supplementary Information for further details).

Acetonitrile is miscible with both chloroform and toluene, but it is a poor solvent for our octapods, so that the addition of acetonitrile to solutions of octapods in either toluene or chloroform causes massive precipitation of the nanocrystals. Mixing of acetonitrile either with the chloroform solution or with a freshly prepared toluene solution led to a precipitate of disordered aggregates of octapods. However, a remarkably different type of precipitate was found when acetonitrile was added to an aged solution of octapods 
in toluene, in which almost all octapods had already assembled into interlocked chains. This precipitate consisted of micrometre-sized 3D superstructures of octapods, together with a fraction of linear chains (Fig. 2a). Several aspects of the sample were peculiar. The isolated chains in this case were longer and straighter than in pure toluene (Supplementary Fig. S2), that is, the chains in the solvent mixture were stiffer (the octapod-octapod distance reduced by $\sim 6 \%$, see Supplementary Information). Furthermore, both the morphology and the internal structure of the ordered 3D assemblies indicated that they were formed through an aggregation process of the linear chains. Given that in some of these clusters entire chains of octapods were missing (Fig. 2b), we conclude that the assembly did not proceed through clustering of individual octapods. We also observed that the nearest-neighbour configuration along one of the principal directions of the crystal was of the same 'interlocked' type as in the single chain (Fig. 2c). The structure could be described with a tetragonal unit cell, with the $c$ direction parallel to the chain axis (see Fig. 2d and Supplementary Information for details).

We established from experiments, and verified using simulations, that pure excluded-volume interactions between the octapods are insufficient to explain chain and superstructure formation. We therefore carried out Hamaker-de Boer type calculations (see Supplementary Information) of the non-retarded vdW forces between octapods based on the shape and the material (CdSe/CdS) of the octapod, in combination with the choice of the medium (toluene/acetonitrile). Several plausible configurations of two octapods were studied, and two of these, the 'interlocked' (Fig. 3b (1)) and the 'pod-pod' parallel configuration (Fig. 3b (2)), were identified as being energetically favourable over the others. Figure $3 \mathrm{~b}$ shows the distance dependence of the vdW interactions for these two configurations. We note that at a distance of twice the length of the capping layer, which is made of surfactants with long hydrophobic alkyl chains, the vdW interaction of these two configurations is similar. At shorter distances, however, the 'interlocked' configuration is more favourable than the 'pod-pod' one, essentially because of the larger capping-layer contact area and the faster decay of the vdW interaction at short range for the pod-pod configuration. Moreover, the 'interlocked' configuration is kinetically more accessible, that is, it is more likely for two octapods to approach each other in solution in a way such that they are in relative orientations close to the interlocked type, rather than in the 'pod-pod' configuration, as the latter requires the two pods of the octapods to be parallel to each other. Simulations with empirical potentials, which roughly model the $\mathrm{vdW}$ potential, showed that chain formation in the dilute liquid phase can indeed be accomplished by longer-ranged attractions between the octahedral cores in combination with short-ranged attractions between the tips of the octapods (see Supplementary Information for further details). A reduced passivation at the tips, due to the local faceted conformation, might also be responsible for a stronger attraction between tips of alternating octapods in the chains. There is clearly ample microscopic justification for our empirical simulation model, which exhibited chain formation as also observed in the experiments.

The formation of chains can be further explained by considering the peculiar geometry of the octapods. In forming a dimer, each octapod can approach another octapod from six possible equivalent directions. However, once a dimer is formed, a third octapod can join and form a stable trimer only by adding to one of the two ends of the chain, which are the only locations where another interlocked configuration can be achieved. Any other side is too sterically hindered by the pods of the other octapods in the dimer. This is shown in the sketch of Fig. 3a. The same holds for the formation of longer chains, realized either by the addition of individual octapods or by gluing of octapod oligomers; that is, linear chains are exclusively formed. On the basis of our a

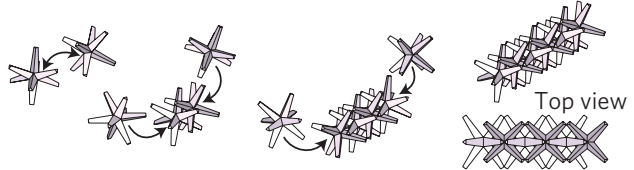

b

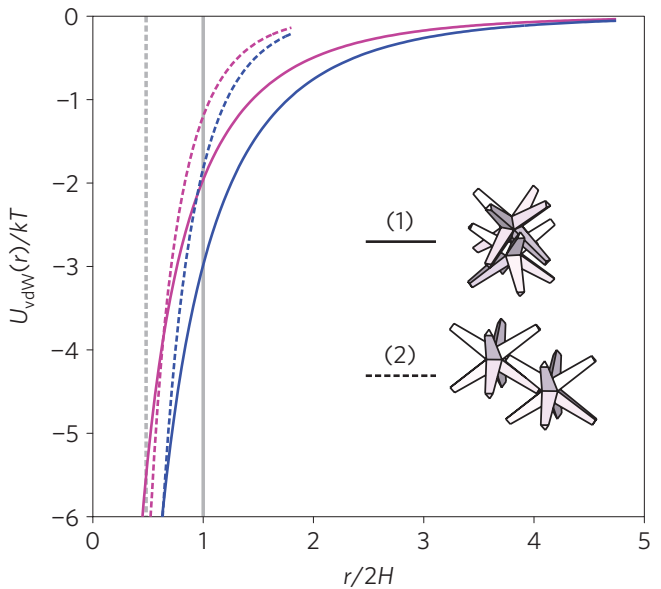

c

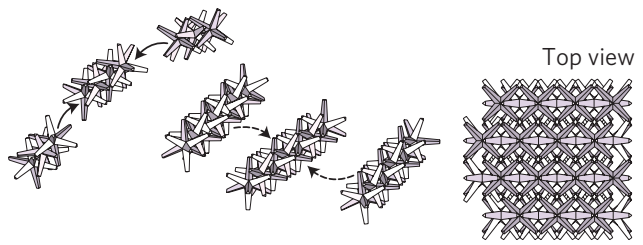

Figure 3 | Model of hierarchical assembly of octapods into 3D structures. a, In the solution, chains are grown by interlocking of octapods, such that in a chain each octapod is tilted by $45^{\circ}$ with respect to its nearest neighbours. b. The vdW attraction can explain both the interlocked configuration seen in the chain (full curve, or (1)), and the 'pod-pod' configuration between two adjacent chains in the superstructure (dashed curve, or (2)), other configurations being less favoured (see Supplementary Information for details). The magenta curves are from a theoretical calculation of the dependence of the vdW interaction strength $U_{\mathrm{vdW}}$ (expressed in units of the thermal energy $k T$, with $k$ Boltzmann's constant and $T$ the temperature) on the separation $r$ between two octapod surfaces, for a pure toluene solvent, whereas the blue curves are for the toluene/acetonitrile (20/80) mixture. The straight lines mark the steric hindrance: the full line considers an ideal capping interaction thickness ( $H$ is the maximum surfactant length), and the dashed line is considered a lower limit. The interlocked configuration between two octapods prevails, explaining the formation of 1D chains as first step. Also note that the interaction strength between octapods in toluene and in the toluene/acetonitrile mixture differs substantially. c, The chains can also grow by on-end interlocking of smaller chains, whereas a 3D superstructure is formed by side-to-side assembly of chains, in this case through 'pod-pod' interactions between octapods belonging to different chains.

analysis we thus conclude that chain formation is driven solely by a combination of the shape, shape-related directional vdW forces, and the dependence of the strength of $\mathrm{vdW}$ forces on the medium. The information for the assembly of these nanocrystals into linear chains is therefore encoded directly in their shape, which bears many analogies with biological systems, for example the self-assembly of collagen proteins into fibrils ${ }^{32}$.

The addition of acetonitrile, a strongly polar solvent, has two effects. First, the effective thickness of the capping layer around each octapod can shrink, because acetonitrile is a poor solvent for its ligands. Second, the increased polarity of the toluene-acetonitrile mixture boosts the vdW interaction strength, see Fig. 3b. Within the confines of our model this has several consequences, in 


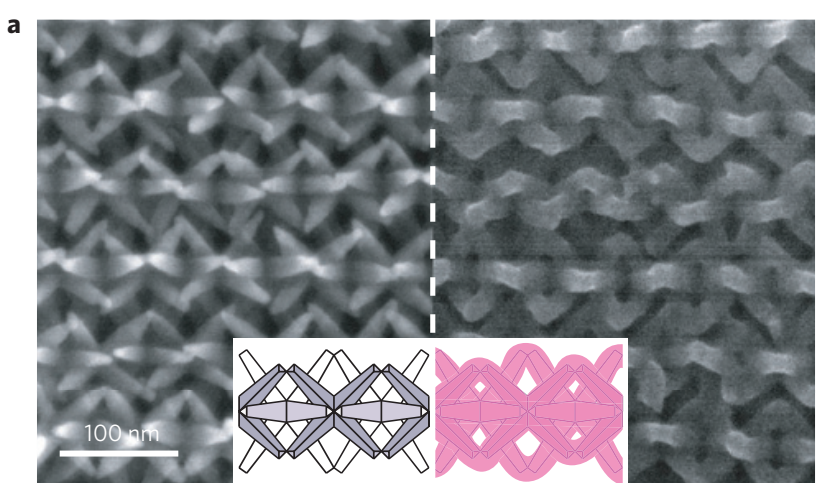

b

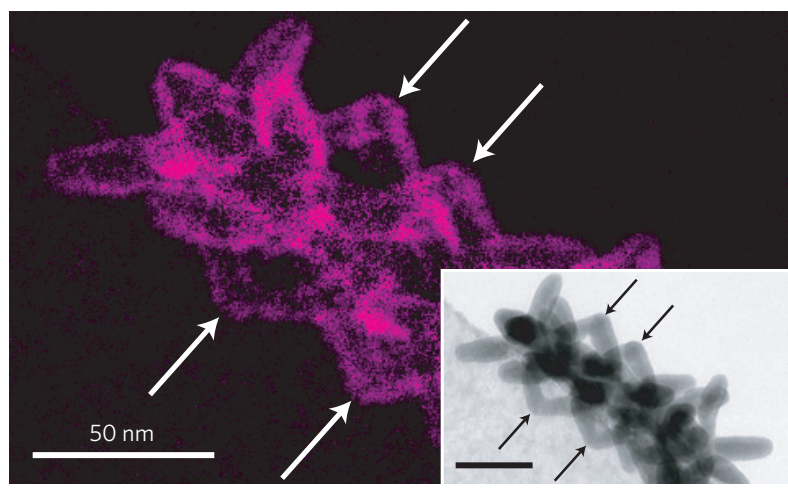

Figure 4 | Welding of the 3D structures. a, The 3D ordered assemblies (left) can be transformed into a welded structure (right), by Cu ion exchange followed by $\mathrm{O}_{2}$ plasma treatment, the octapods that touch at the tips being fused into a nano-link of a chain (as sketched in the insets).

b. The chains after this treatment are welded by a thin shell of copper oxide $(\sim 3 \mathrm{~nm}$ ) surrounding the pods of the octapods, as revealed by EFTEM (the magenta colour is from the oxygen signal, see Supplementary Fig. S7 for details) from a portion of a chain after the same treatment (the inset shows the corresponding TEM image).

agreement with our experimental findings: (1) octapods in pre-existing chains bind more strongly, thereby stiffening these chains; (2) chain-chain interactions become stronger, initially resulting in on-end aggregation, which is of the interlocked type. This initial aggregation is followed by side-by-side aggregation, when the chains are of sufficient length to make such aggregation energetically preferential; (3) the side-by-side aggregation will favour configurations where the pods are in close proximity (see also Supplementary Information), as seen in Fig. 2b,c. A schematic of steps (2) and (3) is given in Fig. 3c to clarify this process. The addition of acetonitrile therefore ultimately yields large, ordered $3 \mathrm{D}$ assemblies with the morphology observed in experiments. These superstructures precipitate when they have grown to sufficient size.

An important feature of these 3D assemblies is the porosity of their structures, enabling the diffusion of reactant species in and out of them. We demonstrate here that, for example, fast cation-exchange reactions can be carried out on the assemblies already deposited on a substrate ${ }^{33}$. By this approach, almost complete exchange occurs quickly and the 3D geometry of the assembly is entirely preserved, as demonstrated in Supplementary Fig. S5, which refers to the same sample as in Fig. $2 \mathrm{c}$ after an almost complete exchange (around $87 \%$ ) of $\mathrm{Cd}^{2+}$ ions with $\mathrm{Cu}^{+}$ions over $15 \mathrm{~min}$ of reaction (see Supplementary Information for details and for cation exchange at longer reaction times). Other reactions, for example controlled oxidation, are possible too: when $\mathrm{Cu}$-exchanged assemblies were subjected to a mild oxygen plasma treatment, a $\mathrm{Cu}_{2} \mathrm{O}$ shell was formed around each octapod (Fig. 4a); the same holding for single chains (Fig. 4b). Even more remarkably, all neighbouring octapods were welded at their tips, as these regions were very close to each other in the starting structures. This welding procedure produced a monolithic, 3D 'nanofabric', very different from an assembly of individual nano-objects, and more akin to a structure prepared by conventional top-down fabrication ${ }^{27,28}$. Important consequences are expected with regards to physical properties such as mechanical strength, which we actually evaluated through a semi-quantitative mechanical characterization (see Supplementary Information for details). Nanoindentation with a cube-corner tip was performed on densely occupied areas of a sample after cation exchange, and after both cation exchange and oxygen plasma treatments, respectively. After the plasma treatment, the load necessary for the indenter tip to cut through the structures rose from $98 \pm 26 \mu \mathrm{N}$ to $124 \pm 40 \mu \mathrm{N}$, that is, a $\sim 25 \%$ increase (uncertainties are all given as standard deviations from the mean values). Moreover, the deep inner layers of the plasma-treated assemblies showed clear signs of welding after these had been exposed using the tip.

Overall, the sequential steps described here, namely synthesis of complex building blocks and their hierarchical assembly and welding, represent an innovative route towards the realization of ordered mesostructures by a pure bottom-up approach. This may lead to several applications: fast (reversible) cation exchange, coupled with the possibility that welded nanostructures might easily conduct charge carriers, might enable the use of such 3D assemblies as ion sensors or as porous electrodes; other applications could arise from the possibility to exploit 3D assemblies of branched nanocrystals of suitable dielectric materials in photonics or plasmonics. Also, mechanical stability could allow the safe transfer of the assemblies to other locations where their spatial positioning could be carefully controlled.

\section{Methods}

Synthesis of the octapods. The octapod-shaped nanocrystals were synthesized according to the procedure described by Deka and colleagues ${ }^{31}$. To achieve the high degree of shape homogeneity and monodispersity reported here, various modifications were made, most notably the relative amounts of chemical precursors and surfactants that were used and various details in the reaction conditions. These are all described in the Supplementary Information.

Formation of the assemblies. A stock solution of octapods in toluene was stored in a nitrogen-filled glove box. As the octapod suspension in toluene is not stable, a precipitate at the bottom of the vial was clearly visible after $24 \mathrm{~h}$; therefore the vial was shaken for a couple of minutes to re-disperse the aggregates. Afterwards, $100 \mu \mathrm{l}$ of this solution were transferred into another empty vial and diluted with the addition of $150 \mu \mathrm{l}$ of toluene. Then the vial was left untouched for $12 / 24 \mathrm{~h}$, after which $1 \mathrm{ml}$ of acetonitrile was added. Again the vial was left untouched for a further $2-5 \mathrm{~h}$, after which a precipitate was seen at the bottom of the vial, and the solution was almost transparent. The wet precipitate was collected with a pipette and transferred onto the conductive substrate for scanning electron microscopy (SEM) or transmission electron microscopy (TEM) analysis. Aggregation in solution was monitored by DLS. The concentration of octapods in the solution was of the order of $10^{-8}$ molar.

SEM and (S)TEM analysis. The samples for SEM and energy dispersive X-ray spectroscopy (EDS) analysis were obtained by depositing a fresh solution on a silicon flat surface, and imaged in a JEOL JSM-7500FA. For cryo-STEM experiments, a vitrified thin film from the solution was obtained by fast plunge-freezing a drop of solution on C-flat holey carbon grids (EMS Quantifoil) into liquid nitrogen, and using a cryo-holder (Gatan 626 cryo-transfer) working at $-180^{\circ} \mathrm{C}$. High angle annular dark field (HAADF) images in STEM mode and energy-filtered transmission electron microscopy (EFTEM) images were acquired on a JEOL JEM-2200FS.

Cation exchange reactions. The CdSe/CdS octapod superstructures, after deposition on the substrate, were dipped in a solution of $37 \mathrm{mg} \mathrm{Cu}\left(\mathrm{CH}_{3} \mathrm{CN}\right)_{4} \mathrm{PF}_{6}$ in $5 \mathrm{ml}$ of methanol for at least $15 \mathrm{~min}$. Afterwards, the substrate with the cation-exchanged superstructures was dipped into $5 \mathrm{ml}$ of pure methanol for $15 \mathrm{~min}$ to wash away excess $\mathrm{Cu}\left(\mathrm{CH}_{3} \mathrm{CN}\right)_{4} \mathrm{PF}_{6}$ and $\mathrm{Cd}$-containing species (released by the cation exchange reaction). This cleaning step was repeated a second time with the addition of another $5 \mathrm{ml}$ of pure methanol. Following this procedure the sample was allowed to dry for several hours.

Oxygen plasma treatment of cation exchanged octapod assemblies. The octapod superstructures, after $\mathrm{Cd}^{2+}$ to $\mathrm{Cu}^{+}$ion exchange, were deposited on the substrate, 
and placed in a Gatan Advanced Plasma System (model Solarus 950). The oxygen plasma was applied for $3 \mathrm{~min}$ at 40 s.c.c.m. $\mathrm{O}_{2}$ flow rate and $25 \mathrm{~W}$ power (13.56 MHz frequency).

Nanoindentation. $10 \times 10$ arrays of indentations on samples were performed in a Micromaterials Nanotest indenter with a cube-corner tip, to a depth of $1 \mu \mathrm{m}$ and with loading and unloading rates of 0.5 and $1 \mathrm{mN} \mathrm{s}^{-1}$, respectively, on samples, either after cation exchange alone or after cation exchange plus oxygen plasma treatment. The indentations of the 3D structures, recognizable from the shape of the load-depth curve, were selected, from which the fracture load (that is, the load reached before the tip penetrates into the silicon substrate) was extracted.

\section{Received 19 May 2011; accepted 16 August 2011; published online 25 September 2011}

\section{References}

1. Quan, Z. W. \& Fang, J. Y. Superlattices with non-spherical building blocks. Nano Today 5, 390-411 (2010).

2. Nie, Z. H., Petukhova, A. \& Kumacheva, E. Properties and emerging applications of self-assembled structures made from inorganic nanoparticles. Nature Nanotech. 5, 15-25 (2010).

3. Li, F., Josephson, D. P. \& Stein, A. Colloidal assembly: The road from particles to colloidal molecules and crystals. Angew. Chem. Int. Ed. 50, 360-388 (2011).

4. Guerrero-Martínez, A., Pérez-Juste, J., Carbó-Argibay, E., Tardajos, G. \& Liz-Marzán, L. M. Gemini surfactant-directed self-assembly of monodisperse gold nanorods into standing superlattices. Angew. Chem. Int. Ed. 48, 9484-9488 (2009).

5. Ciszek, J. W. et al. Assembly of nanorods into designer superstructures: The role of templating, capillary forces, adhesion, and polymer hydration. ACS Nano 4, 259-266 (2010).

6. Baranov, D. et al. Assembly of colloidal semiconductor nanorods in solution by depletion attraction. Nano Lett. 10, 743-749 (2010).

7. Min, Y. J., Akbulut, M., Kristiansen, K., Golan, Y. \& Israelachvili, J. The role of interparticle and external forces in nanoparticle assembly. Nature Mater. 7 , 527-538 (2008).

8. Bishop, K. J. M., Wilmer, C. E., Soh, S. \& Grzybowski, B. A. Nanoscale forces and their uses in self-assembly. Small 5, 1600-1630 (2009).

9. Stebe, K. J., Lewandowski, E. \& Ghosh, M. Oriented assembly of metamaterials. Science 325, 159-160 (2009).

10. Huang, T., Zhao, Q. A., Xiao, J. Y. \& Qi, L. M. Controllable self-assembly of PbS nanostars into ordered structures: Close-packed arrays and patterned arrays. ACS Nano 4, 4707-4716 (2010).

11. Liu, K., Zhao, N. N. \& Kumacheva, E. Self-assembly of inorganic nanorods. Chem. Soc. Rev. 40, 656-671 (2011).

12. Shevchenko, E. V., Talapin, D. V., Kotov, N. A., O’Brien, S. \& Murray, C. B. Structural diversity in binary nanoparticle superlattices. Nature $\mathbf{4 3 9}$ 55-59 (2006).

13. Talapin, D. V.et al. A new approach to crystallization of CdSe nanoparticles into ordered three-dimensional superlattices. Adv. Mater. 13, 1868-1871 (2001).

14. Shevchenko, E. V., Kortright, J. B., Talapin, D. V., Aloni, S. \& Alivisatos, A. P. Quasi-ternary nanoparticle superlattices through nanoparticle design. Adv. Mater. 19, 4183-4188 (2007).

15. Chen, Z. \& O'Brien, S. Structure direction of II-VI semiconductor quantum dot binary nanoparticle superlattices by tuning radius ratio. ACS Nano 2 , 1219-1229 (2008)

16. Evers, W. H., Friedrich, H., Filion, L., Dijkstra, M. \& Vanmaekelbergh, D. Observation of a ternary nanocrystal superlattice and its structural characterization by electron tomography. Angew. Chem. Int. Ed. 48, 9655-9657 (2009).

17. Dong, A. G., Chen, J., Vora, P. M., Kikkawa, J. M. \& Murray, C. B. Binary nanocrystal superlattice membranes self-assembled at the liquid-air interface. Nature 466, 474-477 (2010).
18. Talapin, D. V. et al. CdSe and CdSe/CdS nanorod solids. J. Am. Chem. Soc. 126, 12984-12988 (2004).

19. Evers, W. H. et al. Entropy-driven formation of binary semiconductor-nanocrystal superlattices. Nano Lett. 10, 4235-4241 (2010).

20. Bodnarchuk, M. I., Kovalenko, M. V., Heiss, W. \& Talapin, D. V. Energetic and entropic contributions to self-assembly of binary nanocrystal superlattices: Temperature as the structure-directing factor. J. Am. Chem. Soc. 132, 11967-11977 (2010).

21. Blaak, R., Mulder, B. M. \& Frenkel, D. Cubatic phase for tetrapods. J. Chem. Phys. 120, 5486-5492 (2004).

22. Nykypanchuk, D., Maye, M. M., van der Lelie, D. \& Gang, O. DNA-guided crystallization of colloidal nanoparticles. Nature 451, 549-552 (2008).

23. Park, S. Y. et al. DNA-programmable nanoparticle crystallization. Nature 451, 553-556 (2008)

24. Jones, M. R. et al. DNA-nanoparticle superlattices formed from anisotropic building blocks. Nature Mater. 9, 913-917 (2010).

25. Liu, K. et al. Step-growth polymerization of inorganic nanoparticles. Science 329, 197-200 (2010).

26. Chen, Q., Bae, S. C. \& Granick, S. Directed self-assembly of a colloidal kagome lattice. Nature 469, 381-384 (2011)

27. Li, F., Josephson, D. P. \& Stein, A. Colloidal assembly: The road from particles to colloidal molecules and crystals. Angew. Chem. Int. Ed. 50, 360-388 (2011).

28. Li, F., Yoo, W. C., Beernink, M. B. \& Stein, A. Site-specific functionalization of anisotropic nanoparticles: From colloidal atoms to colloidal molecules. J. Am. Chem. Soc. 131, 18548-18555 (2009).

29. Podsiadlo, P. et al. LBL assembled laminates with hierarchical organization from nano- to microscale: High-toughness nanomaterials and deformation imaging. ACS Nano 3, 1564-1572 (2009).

30. Manna, L., Milliron, D. J., Meisel, A., Scher, E. C. \& Alivisatos, A. P. Controlled growth of tetrapod-branched inorganic nanocrystals. Nature Mater. 2, 382-385 (2003).

31. Deka, S. et al. Octapod-shaped colloidal nanocrystals of cadmium chalcogenides via 'one-pot' cation exchange and seeded growth. Nano Lett. 10, $3770-3776$ (2010).

32. Orgel, J., Irving, T. C., Miller, A. \& Wess, T. J. Microfibrillar structure of type I collagen in situ. Proc. Natl Acad. Sci. USA 103, 9001-9005 (2006).

33. Son, D. H., Hughes, S. M., Yin, Y. D. \& Alivisatos, A. P. Cation exchange reactions-in ionic nanocrystals. Science 306, 1009-1012 (2004).

\section{Acknowledgements}

The authors acknowledge financial support from the European Union through the FP7 starting ERC Grant NANO-ARCH (Contract Number 240111). M.D. acknowledges financial support by a 'Nederlandse Organisatie voor Wetenschappelijk Onderzoek' NWO Vici Grant, and R.v.R. by the Utrecht University High Potential Programme.

\section{Author contributions}

K.M. performed the synthesis of the octapods and optimized the procedures for assembling. D.D. performed DLS measurements and cation exchanged reactions. G.B. performed cryo-STEM experiments, STEM tomographic reconstruction of the chain, and discussed modelling. S.M. imaged all samples with SEM-EDS and had the idea of plasma treatment. R.B. performed EFTEM on welded chains and STEM tomographic acquisitions on single octapods. J.d.G., M.D. and R.v.R. performed all the simulations and discussed modelling. L.C. performed the indentation measurements. L.M. and R.C. initiated the work, had the idea of hierarchical assembling, and discussed modelling.

\section{Additional information}

The authors declare no competing financial interests. Supplementary information accompanies this paper on www.nature.com/naturematerials. Reprints and permissions information is available online at http://www.nature.com/reprints. Correspondence and requests for materials should be addressed to L.M. 